\title{
PLANTAS INTRODUCIDAS EN EL PARQUE NACIONAL TORRES DEL PAINE, CHILE
}

\section{ALIEN PLANTS IN TORRES DEL PAINE NATIONAL PARK, CHILE}

\author{
Erwin Domínguez ${ }^{1}$, Arve Elvebakk ${ }^{2}$, Clodomiro Marticorena ${ }^{3} \&$ Aníbal Pauchard $^{4,5}$ \\ ${ }^{1}$ Centro de Estudios del Cuaternario de Fuego-Patagonia y Antártica (CEQUA). \\ Av. Bulnes 01890, Punta Arenas, Chile. erwindominguez925@ hotmail.com \\ ${ }^{2}$ Department of Biology, Faculty of Science, University of Tromso, N-9037, Norway \\ ${ }^{3}$ Departamento de Botánica, Universidad de Concepción, Casilla 160-C, Concepción. Chile. \\ ${ }^{4}$ Facultad de Ciencias Forestales, Universidad de Concepción, Casilla 160-C, Concepción. Chile. \\ ${ }^{5}$ Instituto de Ecología y Biodiversidad (IEB), Casilla 653, Santiago, Chile.
}

\begin{abstract}
RESUMEN
Se presentan 61 nuevos registros de plantas introducidas para el Parque Nacional Torres del Paine $\left(49^{\circ} 21^{\prime}-51^{\circ} 08^{\prime} \mathrm{S}\right.$ y 730 $07^{\prime}-74^{\circ} 52^{\prime}$ O), Región de Magallanes, con una superficie de 181.414 ha. Se encontraron 85 especies introducidas, pertenecientes a 21 familias y 65 géneros. Las familias mejor representadas en número de especies fueron Poaceae (22 spp.), Asteraceae (11 spp.) y Caryophyllaceae (7 spp.). Las hierbas perennes constituyen el 52\% y las anuales el $34 \%$ de la flora. El 88\% de ellas son de origen europeo y el $36 \%$ corresponde a especies invasoras.
\end{abstract}

Palabras Claves: Plantas no indígenas, plantas invasoras, Reserva de la Biósfera, Parque Nacional Torres del Paine, Chile.

\section{ABSTRACT}

We report 61 new records of alien plants for Torres del Paine National Park. The park is located in the Magellan Region $\left(49^{\circ} 21^{\prime}-51^{\circ} 08^{\prime} \mathrm{S}\right.$ y $\left.73^{\circ} 07^{\prime}-74^{\circ} 52^{\prime} \mathrm{O}\right)$, with an area of 181.414 ha. The study area has 21 families, 65 genera and 85 alien species. The families with the largest number of species are Poaceae (22 spp.), Asteraceae (11 spp.) and Caryophyllaceae (7 spp.).The most important life form is perennial herb, with $52 \%$ of the species and annual herb with $34 \% .88 \%$ of the alien species has a European origin. $36 \%$ species are considered invasive.

Keywords: Non-indigenous plants, invasion species, Biosphere Reserve, Parque Nacional Torres del Paine, Chile.

\section{INTRODUCCION}

Las plantas introducidas han sido reconocidas como un problema potencial para la conservación de áreas protegidas (Lonsdale 1999; Mack et al. 2000, Pauchard \& Alaback 2004) debido a que además de causar la pérdida de diversidad afectan la estructura de los ecosistemas y sus procesos, disminuyendo la capacidad de conservación de las áreas protegidas (Mack et al. 2000). Estudios recientes indican que las invasiones biológicas se están presentando en comunidades estables o hábitats raros o únicos, lo que representa una gran amenaza, puesto que sus avances y efectos son difíciles de predecir (Stohlgren et al. 2001, 2002).

Los estudios en Chile sobre especies de plantas introducidas invadiendo áreas silvestres protegidas se han enfocado en la evaluación de la influencia de los bordes de caminos como vías de dispersión para las invasiones de plantas introducidas, los efectos de la altitud, uso de suelo y los gradientes de disturbio relacionados con el tipo de paisaje (Pauchard \& 
Alaback 2004, Fuentes et al. 2004). Otros estudios han reportado el grado de invasión de plantas introducidas en áreas protegidas. Por ejemplo, en el Parque Nacional Archipiélago de Juan Fernández

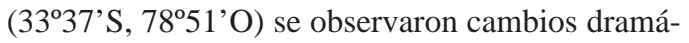
ticos en la composición florística debido a la introducción de plantas exóticas, las que actualmente superan en número a las especies nativas (Matthei et al. 1993, Swenson et al. 1997, Greimler et al. 2002, Cuevas et al. 2004).

En el extremo sur de Chile las especies introducidas no parecen dominar la flora. Por ejemplo, en el Parque Nacional Cabo de Hornos (55 $00^{\circ} \mathrm{S}$, $\left.67^{\circ} 05^{\prime} \mathrm{O}\right)$ se registró un número reducido de especies introducidas (1,8\%)(Rozzi et al. 2004) en comparación a la isla Grande de Tierra del Fuego que ha estado sujeta a constantes disturbios de origen ganadero y forestal (23\%) (Moore \& Goodall 1977, Moore 1983). En la zona continental, sin embargo, para el Parque Nacional Pali Aike $\left(52^{\circ} 03^{\prime} \mathrm{S}\right.$, $69^{\circ} 48^{\prime} \mathrm{O}$ ) la flora introducida representa un $10 \%$ de una flora integrada por un total de 164 especies de plantas vasculares (Domínguez et al. 2004).

Para el Parque Nacional Torres del Paine no existe un listado actualizado de especies introducidas. La primera descripción de la vegetación del parque corresponde al año 1916 cuando éste estaba integrado en su mayor parte por estancias de uso ganadero (Martinic 2000): 63 especies para el área fueron registradas, siendo las primeras especies introducidas reportadas: Cerastium arvense y Galium aparine (Skottsberg 1916); una nueva descripción de la vegetación dio como resultado la presencia de 178 especies nativas y tres especies introducidas (de ellas Poa pratensis corresponde a un nuevo registro para el parque) (Pisano 1974). El bajo número de especies exóticas en este estudio es debido a que el autor no consideró los sitios perturbados por los incendios y la actividad ganadera dentro del parque.

Boelcke et al. 1985 señalan para el parque 85 especies, de ellas nueve especies son introducidas y seis corresponden a nuevos registros (Agrostis capillaris, Aira caryophyllea, Aira praecox, Cerastium fontanum, Plantago lanceolata y Veronica serpyllifolia). En estudios relacionados con los hábitats de alimentación de los guanacos (Lama guanicoe) se reportaron cuatro nuevas especies introducidas (Holcus lanatus, Potentilla anserina, Arrhenatherum elatius y Trifolium repens) presentes en vegas (Ortega \& Franklin1988). Dollenz
1991 a reporta la presencia de la especie introducida Rumex acetosella y su capacidad de invadir áreas perturbadas por un incendio dentro del parque. Estudios que describen las diferentes etapas de sucesión vegetal en morrenas del glaciar Dickson informan la presencia de 36 especies nativas y 2 introducidas (Dollenz 1991b). Para la flora alpina de la cordillera del Paine se encontraron sólo dos especies introducidas de un total de 177 especies registradas para el área (Arroyo et al. 1992). En análisis de pastizales incendiados en el sector del glaciar Grey se registró un total de 45 especies nativas y 11 introducidas, siendo seis de ellas nuevas para el parque (Dollenz \& Ivanovic 1996). Por otro lado, la evaluación de la composición florística de sitios recientemente desglaciados en el sector del glaciar Tindall aportó un total de 135 especies de plantas nativas y 8 introducidas, siendo tres de ellas nuevos registros para el parque (Pisano et al. 1997, Henríquez 2002). El último aporte corresponde a Conium maculatum, especie exótica que ha adquirido importancia en los bordes de camino dentro del parque (Domínguez 2003). En resumen, el total de especies introducidas reportadas entre los años 1916 y 2003 es de 24 especies para el parque, cifra que puede ir en aumento como resultado de los disturbios generados por el incremento de la actividad antrópica (e.g., aumento de incendios, infraestructura hotelera, caminos, senderos y número de visitas).

El objetivo de este trabajo es dar a conocer un inventario de las plantas introducidas que se encuentran dentro del Parque Nacional Torres del Paine, junto con evaluar la forma de vida, el origen y status de invasión de cada taxón reportado. Esta información podrá ser utilizada para las acciones de restauración y manejo de este parque, especialmente en aquellas áreas afectadas por el incendio del año 2005.

\section{MATERIALES Y METODOS}

Area de ESTudio

El Parque Nacional Torres del Paine $49^{\circ} 21^{\prime}-51^{\circ} 08^{\prime} \mathrm{S}$ y $73^{\circ} 07^{\prime}-74^{\circ} 52^{\prime} \mathrm{O}, 25$ - 3.050 m s.n.m. (Figura 1), ubicado en la provincia de Ultima Esperanza (Región de Magallanes, Chile), fue reconocido internacionalmente en 1978 por la UNESCO como Reserva de la Biosfera, siendo su principal función la conservación de paisajes, ecosistemas, especies y 
Plantas introducidas, Torres del Paine: Domínguez, E. ET AL.

diversidad genética (Benoit 2005). Es uno de los más emblemáticos de Chile y el más importante de la región en términos turísticos y económicos, con un crecimiento sostenido en el tiempo de visitantes extranjeros y nacionales superior a las 100.000 visitas. Su superficie es de 181.414 ha (Benoit 2005) y se caracteriza por su heterogeneidad paisajística, en donde convergen montañas, glaciares, valles, humedales y grandes lagos.
El clima al noroeste del parque es afectado por el campo de hielo sur de la Patagonia y las altas cadenas montañosas de la Cordillera Paine que se levantan hasta los 3050 m s.n.m. Al sureste predominan las colinas con suaves pendientes hasta llegar a las llanuras. La vegetación se distribuye en cuatro zonas bióticas: estepa patagónica, matorral preandino, bosque deciduo de Magallanes y Desierto Andino (Pisano 1974).

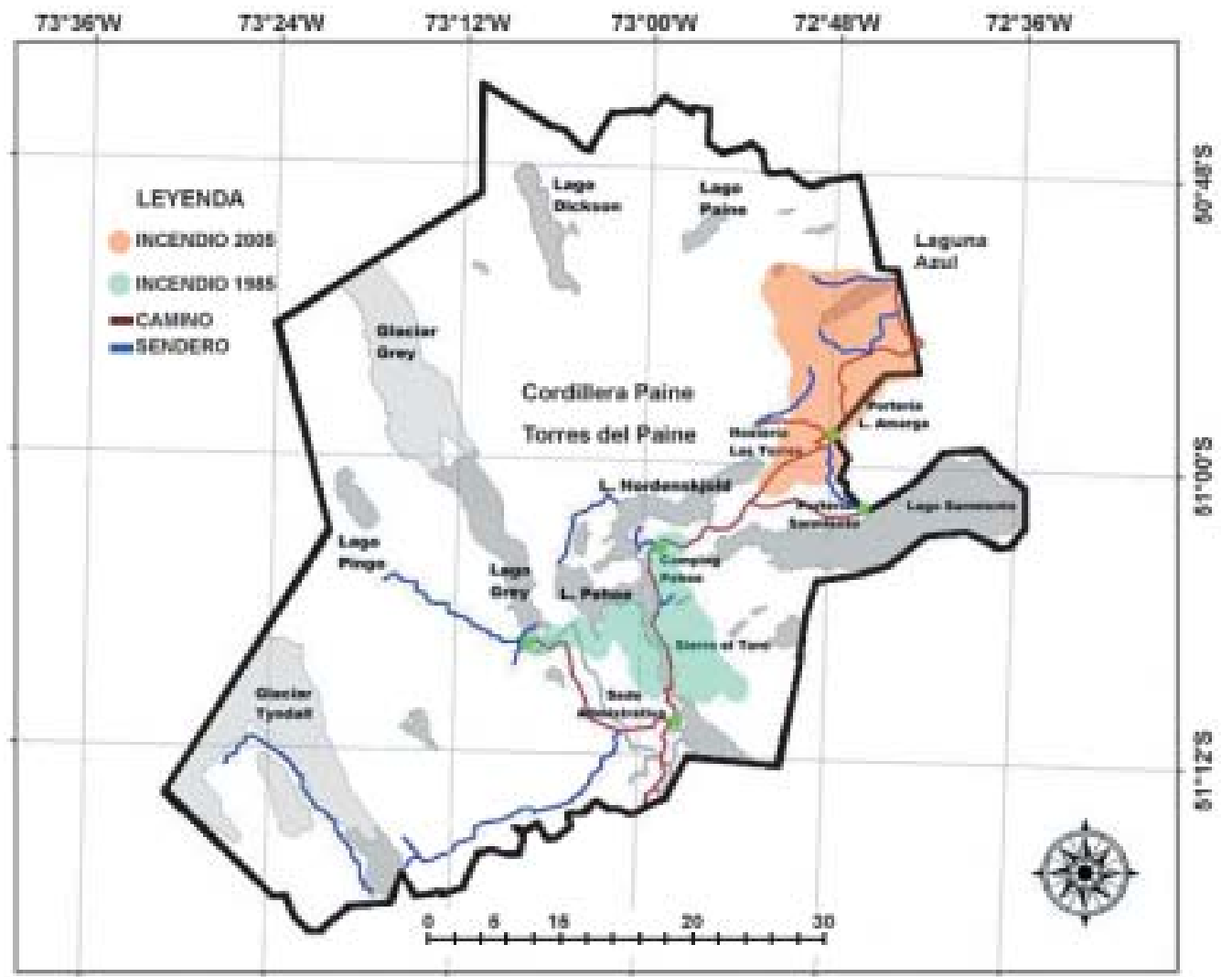

Figura 1. Mapa de áreas incendiadas (1985-2005), caminos, senderos e hitos geográficos dentro del Parque Nacional Torres del Paine, Región de Magallanes, Chile.

FiguRE 1. Map of burnt areas (1985-2005), roads, trails and geographic points in Torres del Paine National Park, Magellan Region, Chile. 
MÉTOdos

Este trabajo está basado en quince viajes de colección entre 1994 y 2006. Se recolectó un total aproximado de 816 ejemplares en los principales tipos de vegetación siguiendo la gradiente de precipitación oeste-este, la cual está asociada con la diversidad taxonómica y la riqueza florística del parque (Arroyo et al. 1992). Además se realizaron colectas en los bordes de los caminos principales y senderos recorriendo un total de $171 \mathrm{~km}$ dentro del parque y las dos principales áreas incendiadas. La primera, formada por el sector Lago Grey, Lago Pehoe y Sierra del Toro que corresponden al incendio de 1985 que afectó un total de 12.983 ha; y la segunda, que corresponde al incendio ocurrido el 17 de febrero de 2005, que afectó 11.685 ha, principalmente al ecosistema de la estepa patagónica (70\%), comprometiendo pastizales y numerosos matorrales; entre ellos destaca los de mata negra formado por las especies con problemas de conservación Junellia tridens, Adesmia boronioides, A. volckmannii y Alstroemeria patagonica, siendo esta comunidad única en su tipo dentro de un área silvestre protegida en Chile ubicada en el sector de Laguna Amarga (Figura 1). La información fue complementada con la revisión de 1.962 ejemplares depositados en el herbario de la Universidad de Concepción (CONC) y colecciones depositadas en el herbario del Instituto de la Patagonia (HIP) que corresponden a plantas colectadas en el Parque Nacional Torres del Paine (PNTP) entre 1945 y 2005. La identificación se basó en Correa (1971-1999), Matthei (1995) y Moore (1983). La nomenclatura sigue a Cronquist (1981) y las actualizaciones mantenidas por la Base de Datos del Proyecto Flora de Chile del herbario de la Universidad de Concepción (Marticorena, datos no publicados). Las especies fueron clasificadas de acuerdo a su ciclo y forma de vida en hierbas anuales, bienales y perennes, arbustos, árboles, y de acuerdo a su origen geográfico. Además se evaluó el status de invasión basado en la clasificación de Pysek et al. (2004). El material herborizado fue depositado en CONC y HIP.

TABla I. Distribución del número de géneros y especies introducidas por familias presentes en el Parque Nacional Torres del Paine (Región de Magallanes).

TABLE I. Distribution of the number of alien genera and species in Torres del Paine National Park, Magellan Region, Chile.

\begin{tabular}{lcc}
\hline Familia & $\mathrm{N}^{\mathbf{o}}$ de géneros & $\mathrm{N}^{\mathbf{0}}$ de especies \\
\hline Poaceae & 14 & 22 \\
Asteraceae & 11 & 11 \\
Caryophyllaceae & 5 & 7 \\
Brassicaceae & 6 & 6 \\
Fabaceae & 4 & 5 \\
Scrophulariaceae & 2 & 4 \\
Polygonaceae & 2 & 4 \\
Apiaceae & 3 & 3 \\
Lamiaceae & 3 & 3 \\
Geraniaceae & 2 & 3 \\
Boraginaceae & 1 & 3 \\
Rosaceae & 2 & 2 \\
Salicaceae & 2 & 2 \\
Plantaginaceae & 1 & 2 \\
Chenopodiaceae & 1 & 2 \\
Papaveraceae & 1 & 1 \\
Caprifoliaceae & 1 & 1 \\
Cupressaceae & 1 & 1 \\
Ranunculaceae & 1 & 1 \\
Rubiaceae & 1 & 1 \\
Urticaceae & 1 & 1 \\
\hline Total & 65 & 85 \\
\hline
\end{tabular}




\section{RESULTADOS}

Se reporta 61 nuevas adiciones para flora introducida del PNTP, de ellas cuatro nuevas para la Región de Magallanes (Geranium molle, Petrorhagia dubia, Geranium pusillum y Poa bulbosa), dando origen a un total de 85 especies, 65 géneros y 21 familias, de las cuales 19 son Magnoliopsida (Dicotyledoneae), una Liliopsida (Monocotyledoneae) y una Gymnospermae (Tablas I y V). Las familias mejor representadas fueron Poaceae (22 especies), Asteraceae (11) y Caryophyllaceae (7); 8 familias con 6 a 3 especies y 10 familias con dos a una especies, las que representan el 19\% de la riqueza total. Los géneros mejor representados fueron: Poa, Cerastium, Myosotis, Rumex, Veronica, Aira, Alopecurus, Bromus, Chenopodium, Geranium, Plantago. Los géneros representados por una especie fueron 53 en total (Tabla II).

Las hierbas perennes son la forma de vida más abundante con 44 especies (52\%) adquiriendo importancia en bermas de caminos y en senderos: Rumex acetosella en la estepa patagónica y en matorrales preandinos; Dactylis glomerata, y Poa pratensis en bosques incendiados de Nothofagus antarctica, y $N$. pumilio en el sector del Lago Pehoe en 1985. Tripleurospermum maritimum es frecuente en bosques achaparrados de $N$. antarctica recientemente incendiados en el sector de la Hostería las Torres (Domínguez, datos no publicados). Las hierbas anuales representaron un $34 \%$ del espectro de formas de vida (Tabla III); entre ellas destacan: Erodium cicutarium y Draba verna, siendo ambas importantes en términos de frecuencia y cobertura especialmente en matorrales de Junellia tridens recientemente incendiados en el sector de Laguna Amarga (Domínguez, datos no publicados).

De las 85 especies introducidas que crecen en el parque, 75 (88\%) son de origen europeo, cuatro euroasiáticas, dos asiáticas, dos de Norteamérica, una de Sudamérica y una Americana (Tabla IV). Entre las primeras destaca Conium maculatum, la cual se ha convertido en una de las especies más peligrosas dentro del parque al utilizar los caminos principales (portería Laguna Amarga-Administración) como un eficiente corredor de dispersión en menos de 10 años. Una situación similar ocurre con la especie euroasiática Lupinus polyphyllus var. polyphyllus que crece en las bermas de caminos y en los jardines de la administración de CONAF, aumentando significativamente el número de individuos durante los últimos nueve años.

TABLA II. Distribución del número de especies introducidas para los géneros presentes en el Parque Nacional Torres del Paine (Región de Magallanes).

TABLE II. Distribution of the number of alien species for the genera present in Torres del Paine National Park, Magellan Region, Chile.

\begin{tabular}{lc}
\hline Género & $N^{o}$ de especies \\
\hline Poa (Poaceae) & 4 \\
Cerastium (Caryophyllaceae) & 3 \\
Myosotis (Boraginaceae) & 3 \\
Veronica (Scrophulariaceae) & 3 \\
Rumex (Polygonaceae) & 3 \\
Aira (Poaceae) & 2 \\
Alopecurus (Poaceae) & 2 \\
Bromus (Poaceae) & 2 \\
Chenopodium (Chenopodiaceae) & 2 \\
Geranium (Geraniaceae) & 2 \\
Plantago (Plantaginaceae) & 2 \\
Trifolium (Fabaceae) & 2 \\
Vulpia (Poaceae) & 2 \\
Géneros con una especie & 53 \\
\hline
\end{tabular}


Tabla III. Distribución del número de especies introducidas y porcentaje por forma de vida en el Parque Nacional Torres del Paine (Región de Magallanes).

TABLE III. Distribution of the number of alien species and percentage by life forms in Torres del Paine National Park, Magellan Region, Chile.

\begin{tabular}{lcc}
\hline Forma de vida & $\mathrm{N}^{\circ}$ de especies & $\%$ \\
\hline Hierba perenne (H) & 44 & 52 \\
Hierba anual (A) & 29 & 34 \\
Arboles (T) & 5 & 6 \\
Hierba Anual /Bienal (AB) & 4 & 5 \\
Hierba Bienal (B) & 2 & 2 \\
Arbusto (S) & 1 & 1 \\
\hline
\end{tabular}

TABLA IV. Origen geográfico de las plantas introducidas presentes en el Parque Nacional Torres del Paine (Región de Magallanes).

TABLE IV. Geographic origin of the alien plants in Torres del Paine National Park, Magellan Region, Chile.

\begin{tabular}{lcc}
\hline Origen geográfico & $\mathrm{N}^{\mathrm{o}}$ de especies & $\%$ \\
\hline Europa & 75 & 88 \\
Euroasiático & 4 & 5 \\
Asia & 2 & 2 \\
Norteamérica & 2 & 2 \\
Sudamérica & 1 & 1 \\
América & 1 & 1 \\
\hline
\end{tabular}

De las 85 especies introducidas 31 (36\%) son clasificadas (sensu Pysek et al. 2004) como especies invasoras, entre las que destacan: Plantago lanceolata (siete venas), Rumex acetosella (vinagrillo), Taraxacum officinale (diente de león), Cerastium arvense (cerastio) Tripleurospermum maritimum subsp. inodorum, Conium maculatum (cicuta), Dactylis glomerata (pasto ovillo) y Poa pratensis, por encontrarse poblaciones de 50 individuos por $1000 \mathrm{~m}^{2}$ en un número superior de 10 localidades distante cada una de ellas $5 \mathrm{~km}$, en hábitats naturales (e.g., matorrales de Mulinum spinosum, en coironales de Festuca gracillima, en matorrales de Gaultheria mucronatay, Junellia tridens entre otros); seminaturales como en los bordes de senderos y zonas de camping, adquiriendo especial importancia en sitios incendiados dentro del parque, como son: Laguna Azul, Sierra el Toro, sector Pehoe, Lago Grey y Laguna Amarga. Las especies naturalizadas son 27 , con una distribución más restringida, las otras restantes son clasificadas como casuales, entre ellas encontramos plantas leñosas que han sido plantadas con fines ornamentales y especialmente como cortaviento antes de que el parque existiera (Cupressus macrocarpa; Laburnum anagyroides, Sorbus aucuparia, Salix viminalis y Populus nigra). Ninguna de estas especies ha podido traspasar la barrera reproductiva (Tabla V).

\section{DISCUSION}

Las familias Poaceae (26\%), Asteraceae (13\%), Caryophyllaceae $(8 \%)$, Brassicaceae $(7 \%)$ y Fabaceae (6\%) comprenden el 54\% de especies introducidas, siendo un valor alto pero coincidente con lo descrito para Chile central (Pauchard \& Alaback 2004). Esta tendencia, en el caso de la familia Poaceae, no sorprende porque los ganaderos que rodean al PNTP siempre se han mostrado interesados en aumentar la calidad nutritiva de sus pasturas a través de la siembra de plantas forrajeras especialmente gramíneas (e.g. Dactylis glomerata, Holcus lanatus, Arrenatherum elatius var. bulbosus, Bromus hordeaceus, Phleum pratense, entre otras). Actualmente, estas gramíneas no requieren la asistencia humana para su propagación, encontrándose individuos fértiles y con semillas fuera y dentro del parque.

Entre las formas de vida las hierbas anuales representaron un $35 \%$ de la flora introducida, siendo éstas un 13\% más que las reportadas para el Parque Nacional Pali Aike (Domínguez et al. 2004). Seguramente, esta diferencia se debe a condiciones climáticas más favorables especialmente en el sector de la estepa patagónica en el Parque Nacional Torres del Paine, el cual se caracteriza por deshielos tempranos en primavera y una lenta desecación que ocurre entre agosto y noviembre, lo cual favorecería una mayor diversidad de esta forma de vida. Sin embargo, cualquier explicación para esto necesita ser probada con estaciones climáticas.

El alto número de especies de origen europeo $(88 \%)$ estaría relacionado con los inmigrantes extranjeros que se establecieron entre los años 18881902 dentro y alrededor del parque (Martinic 2000), 
introduciendo plantas forrajeras de regiones mediterráneas para aumentar la producción de ganado; estos resultados son consistentes con estudios realizados en el Parque Nacional Villarrica y Huerquehue (Pauchard \& Alaback 2004) y en Norteamérica (McKinney 2002), indicando un paralelismo entre la colonización europea, la historia ganadera y las invasiones.

La intensidad y esfuerzo de muestreo realizado en este estudio a lo largo de caminos, senderos y sitios incendiados mostró un alto número de especies introducidas, las que estarían relacionadas con los incendios forestales, los que parecen ser el principal disturbio; así lo demuestran los 28 siniestros ocurridos entre 1985-2005, afectando el $17 \%$ (30.246 ha) de la superficie total del Parque Nacional Torres del Paine (datos proporcionados por CONAF-Magallanes). Los incendios forestales, al parecer, han generado las condiciones ambientales ideales para la propagación de especies invasoras como Rumex acetosella, Taraxacum officinale, Plantago lanceolata y Cerastium arvense, las cuales han invadido casi todos los hábitats presentes dentro del parque, incluso llegando a formar parte de la flora alpina y colonizando sitios recientemente desglaciados como ha sido reportado para los glaciares Dickson y Tindall (Arroyo et al. 1992, Dollenz 1991a, Dollenz \& Ivanovic 1996, Pisano et al. 1997, Henríquez 2002).

Finalmente, debemos considerar que las invasiones biológicas pueden estar modificando la estructura y composición de las comunidades vegetales del Parque Nacional Torres del Paine, como ha sido detectado en otras áreas protegidas a nivel mundial y nacional (D’Antonio \& Vitousek 1992; Lonsdale 1999; Mack et al. 2000; Cuevas et al. 2004; Pauchard \& Alaback 2004). Es fácil comprender entonces la conveniencia que hay en controlar los ac- tuales focos de invasión y limitar fuertemente la introducción de nuevas especies exóticas en uno de los parque nacionales y Reserva de la Biosfera más importante de la Patagonia austral y de Chile.

Para lograr estos objetivos es necesario elaborar un plan de manejo de especies introducidas específico para el PNTP que incluya: (1) eliminación completa del ganado doméstico dentro del parque (Pauchard \& Alaback 2004); (2) control sistemático de la vegetación exótica mediante la elaboración de mapas de distribución, proporcionando éstos la información básica para planificar la eliminación o control de especies introducidas, de acuerdo a su grado de agresividad (Byers et al.2002; Pauchard $\&$ Alaback 2004); (3) establecer un plan de acciones de restauración de las comunidades vegetales nativas en zonas afectadas por perturbaciones naturales o antrópicas. Para lograr estas acciones es necesario incorporar a todas las entidades que están vinculadas al PNTP, incluyendo al personal de hoteles, hosterías y guías de turismo, para poder desarrollar una red de monitoreo que permita prevenir nuevas invasiones biológicas (e.g., Hieracium pilosella) que podrían afectar los procesos evolutivos y el desarrollo turístico en forma negativa en los próximos años.

\section{AGRADECIMIENTOS}

Se agradece a la Corporación Nacional Forestal (CONAF) XII Región, a la Hostería las Torres por las facilidades otorgadas para el trabajo de campo. También al personal del Herbario de la Universidad de Concepción CONC y al Herbario del Instituto de la Patagonia de la Universidad de Magallanes HIP y al Centro de Estudios del Cuaternario (CEQUA). 
Gayana Bot. 63(2), 2006

TABLA V. Lista de las especies introducidas para el Parque Nacional Torres del Paine por categoría taxonómica, considerando: la forma de vida $(\mathrm{A}=$ Hierba Anual; $\mathrm{B}=\mathrm{Hierba} \mathrm{Bienal}$; $\mathrm{H}=$ Hierba Perenne; $\mathrm{S}=$ Arbusto; $\mathrm{T}=\mathrm{Arbol})$; Origen $(\mathrm{EU}=$ Europa; EA= Eurasiática; A= Asiática; AN= América del Norte; AS= América del Sur); Status de invasión $(\mathrm{C}=\mathrm{Casual}$; $\mathrm{N}=$ Naturalizada; I= Invasora). * Nueva para el Parque Nacional Torres del Paine, ** Nueva para Magallanes.

TABLE V. List of the alien species in Torres del Paine National Park by taxonomic category, considering the life form $(\mathrm{A}=$ Annual herb; $\mathrm{B}=$ Biannual herb; $\mathrm{H}=$ Perennial herb; $\mathrm{S}=\mathrm{Shrub}$; $\mathrm{T}=$ Tree); Origin (EU= Europe; EA= Eurasia; $\mathrm{A}=\mathrm{Asia}$; $\mathrm{AN}=$ North America; $\mathrm{AS}=$ South America); Invasion status $(\mathrm{C}=\mathrm{Casual} ; \mathrm{N}=$ Naturalized; $\mathrm{I}=$ Invasive $)$ * New for Torres del Paine National Park, ** New for Magellan.

\title{
GYMNOSPERMAE \\ Pinopsida
}

CUPRESSACEAE

1. T-AN-N Cupressus macrocarpa Hartw. ex Gordon *

\section{MAGNOLIOPHYTA}

Magnoliopsida (Dicotyledoneae)

\author{
APIACEAE \\ 2. AB-EU-I \\ 3. H-EU-C \\ Conium maculatum L. \\ Daucus pusillus Michx.* \\ 4. H-EU-C Levisticum officinale Koch.* \\ ASTERACEAE \\ 5. H-EU-I \\ 6. H-EU-C \\ 7. H-EU-C \\ 8. A-AN-I \\ 9. A-EU-I \\ 10. A-EU-N \\ 11. H-EU-N \\ 12. H-EU-I \\ 13. H-EU-I \\ 14. H-EU-I \\ 15. AB-EU-C \\ Achillea millefolium L.* \\ Artemisia absinthium L.* \\ Bellis perennis L. * \\ Matricaria discoidea DC.* \\ Cirsium vulgare (Savi) Ten.* \\ Crepis capillaris (L.) Wallr. * \\ Hypochaeris radicata $\mathrm{L}$. \\ Leucanthemum vulgare L.* \\ Taraxacum officinale Weber ex F.H.Wigg. \\ Tripleurospermum maritimum (L.) W.D.J.Koch subsp. inodorum (L.) Appleq.* \\ Sonchus asper (L.) Hill* \\ BORAGINACEAE \\ 16. B-EU-C \\ 17. A-EU-N \\ Myosotis arvensis (L.) Hill * \\ Myosotis discolor Pers. subsp. discolor \\ 18. H-EU-I \\ Myosotis stricta Link ex Roem. et Schult. * \\ BRASSICACEAE \\ 19. A-EU-N \\ 20. A-EU-I \\ Alyssum alyssoides (L.) L.* \\ Brassica rapa $\mathrm{L} . *$ \\ Capsella bursa-pastoris (L.) Medik.* \\ 21. AB-EU-I \\ Descurainia sophia (L.) Webb ex Prantl \\ 23. A-EU-I \\ Draba verna $\mathrm{L}$. \\ 24. A-A-N \\ Lepidium didymum $\mathrm{L}$.* \\ CAPRIFOLIACEAE \\ 25. S-EA-C Sambucus nigra L.* \\ CARYOPHYLLACEAE \\ 26.H-EU-N Arenaria serpyllifolia L.* \\ 27. H-EA-I Cerastium arvense $\mathrm{L}$. \\ 28. A-EU-C Cerastium fontanum Baumg. subsp. vulgare (Hartman) Greuter et Burdet \\ 29. A-EU-N Cerastium glomeratum Thuill.*
}


Plantas introducidas, Torres del Paine: Domínguez, E. ET AL.

Continuación Tabla V

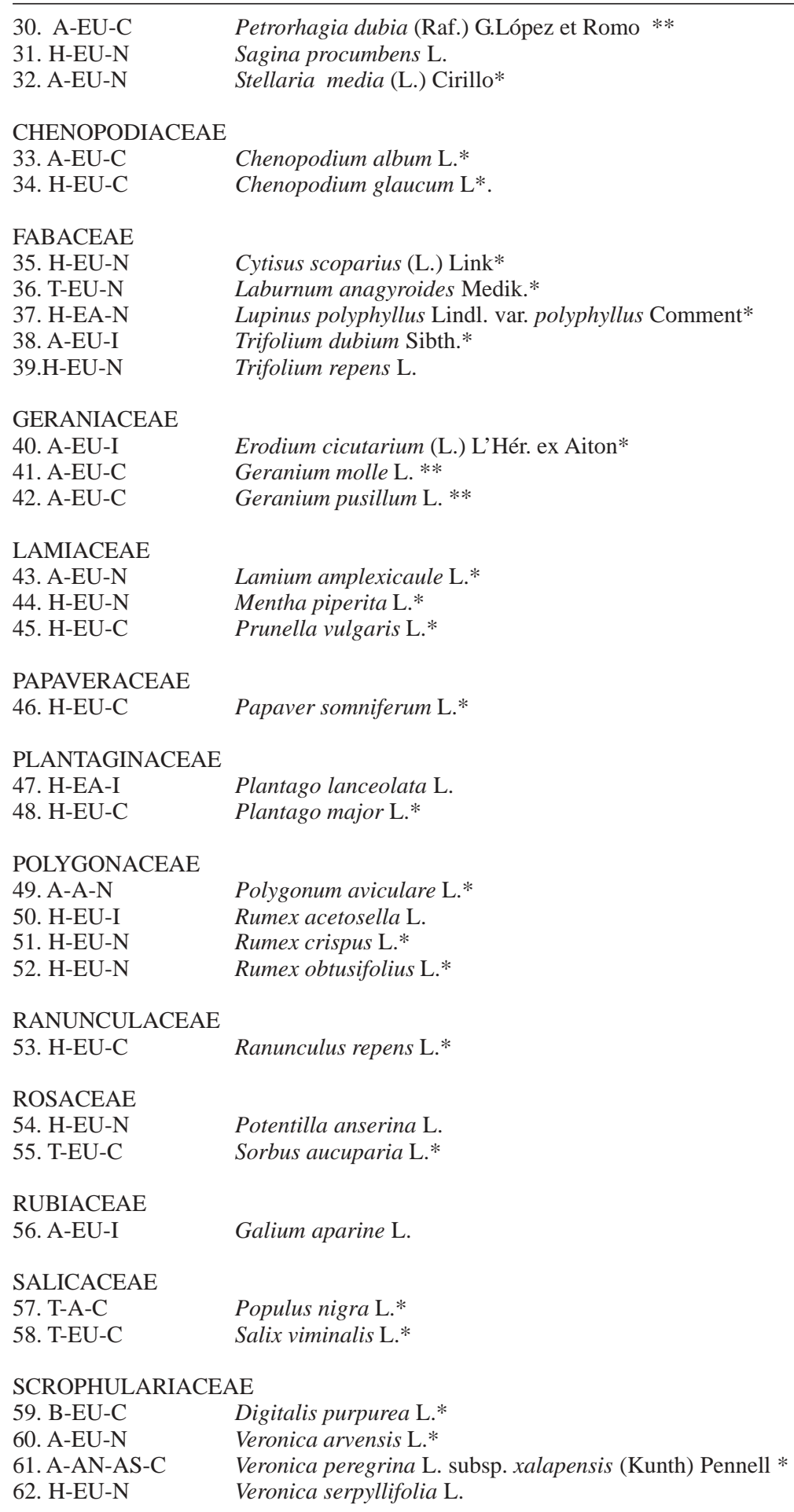


Gayana Bot. 63(2), 2006

Continuación Tabla V

URTICACEAE

63. A-EU-N

POACEAE

64. H-EU-N

65. A-EU-N

66. A-EU-N

67. H-EU-C

68. H-EU-N

69. H-EU-C

70. H-EU-N

71. A-EA-C

72. H-EU-C

73. H-EU-I

74. H-EU-C

75. H-EU-N

76. A-EU-N

77. A-EU-N

78. H-EU-N

79. H-EU-N

80. A-EU-C

81. H-EU-C

82. H-EU-I

83. H-EU-N

84. A-EU-C

85. A-EU-N
Urtica urens L.*

Liliopsida (Monocotyledoneae)

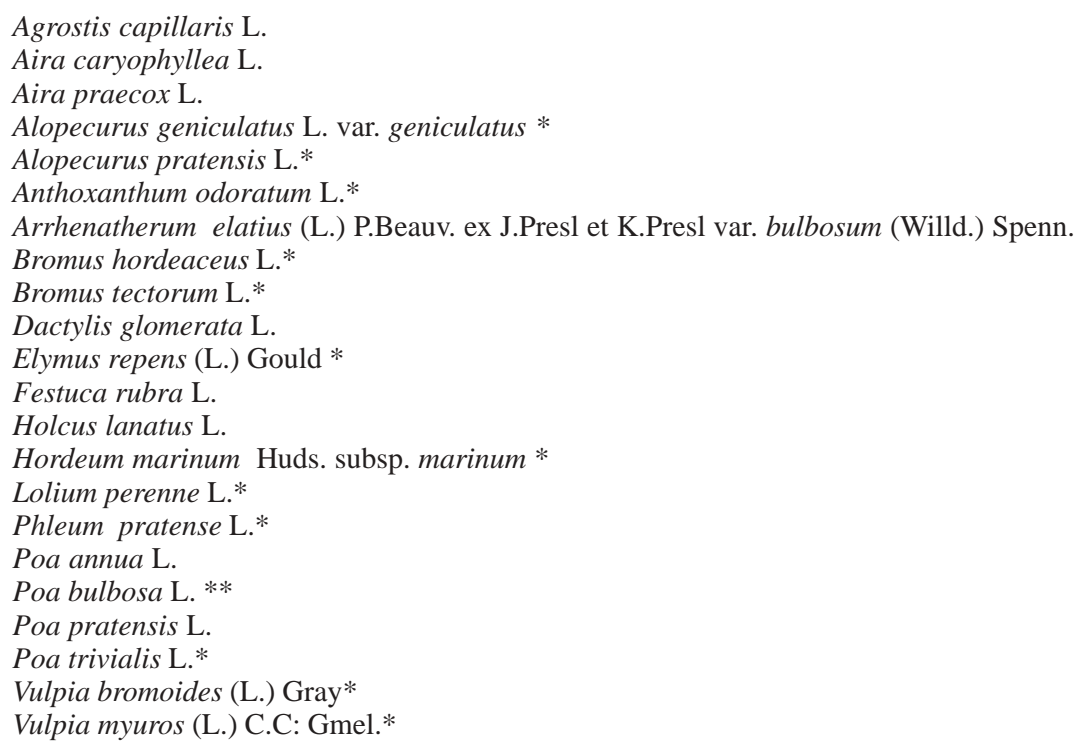

\section{BIBLIOGRAFIA}

Arroyo, M.T.K., C.P. von Bohlen, L. Cavieres \& C. Marticorena. 1992. Survey of the flora of Torres del Paine National Park, Chile. Gayana Botánica. 49(1-4):47-70.

Benoit, I. 2005. Areas Silvestres Protegidas del Estado: una visión histórica. Revista Chile Forestal N³12. Documento Técnico 162:1-12.

Boelcke, O., D.M. Moore \& F.A. Roig (Eds.). 1985. Transecta Botánica de la Patagonia Austral. Consejo Nacional de Investigación Científica y Técnica (Argentina). Buenos Aires. XXVIII, 733 pp.

Byers, J.E., S. Reichard, J.M. Randall, I.M. Parker, C.S. Smith, W.M. Lonsdale, I.A.E. AtKinson, T.R. Seastedt, M. Williamson, E. Chornesky \& D. HAYES. 2002. Directing research to reduce the impacts of nonindigenous species. Conservation Biology 15:91-97.

Correa, M.N. (Ed.). 1971-1999. Flora Patagónica. Partes I-VI. Colección Científica del INTA, Tomo VIII. Buenos Aires.

Cronquist, A. 1981. An integrated system of classification of flowering plants. Columbia University Press, New York. 1262 pp.

Cuevas, J., A. Marticorena \& L. Cavieres. 2004. New additions to the introduced flora of the Juan Fernández Islands: origin, distribution, life history traits, and potential of invasion. Revista Chilena de Historia Natural 77: 523-538.

D’Antonio, C.D. \& P.M. VitouseK. 1992. Biological invasions by exotic grasses, the grass/fire cycle, and global change. Annual Review of Ecology and Systematics 23:63-87.

Dollenz, O. 1991a. Capacidad de colonización de Rumex acetosella $\mathrm{L}$. en comunidades perturbadas. Anales Instituto Patagonia, Serie Ciencias, Naturales, Punta Arenas (Chile), 20 (1):61-67.

Dollenz, O. 1991b. Sucesión vegetal en el sistema morrénico del glaciar Dickson, Magallanes, Chile. Anales Instituto Patagonia, Serie Ciencias, Naturales, Punta Arenas (Chile) 20(1): 49-60.

Dollenz, O. \& J. Ivanovic. 1996. Sucesión secundaria en un pastizal incendiado en el Parque Nacional Torres del Paine, Magallanes, Chile. Anales Instituto Patagonia, Serie Ciencias, Naturales, Punta Arenas (Chile), 24:15-28.

Domínguez, E. 2003. Orquídeas del Parque Nacional Torres del Paine. Revista Chile Forestal $N^{\circ} 300$. Documento Técnico 150:1-11.

Domínguez, E., C. Marticorena, A. Elvebakk \& A. Pauchard. 2004. Catálogo de la flora vascular del 
Parque Nacional Pali Aike. XII Región, Chile. Gayana Botánica 61(2):67-72.

Fuentes, N., E. Ugarte \& S. KLOtZ. 2004. Flora asociada a bordes de camino en un transecto este-oeste en la VIII Región, Chile. Boletín del Museo Nacional de Historia Natural, Chile, 53:37-49.

Greimler, J., P. López, T.F. Stuessy, T. Dirnböck. 2002. The vegetation of Robinson Crusoe Island (Isla Masatierra) Juan Fernández Archipelago, Chile. Pacific Science 56:263-284.

Henríquez, J.M. 2002. Análisis de la flora vascular de valles glaciares de la Región de Magallanes, Chile. Anales Instituto Patagonia, Serie Ciencias, Naturales (Chile) 30:25-40.

LONSDALE, W.M. 1999. Global patterns of plant invasions, and the concept of invasibility. Ecology 80:15221536.

Mack, R.N., D. Simberloff, W.M. Lonsdale, H. Evans, M. Clout \& F. A. Bazzaz. 2000. Biotic invasions: Causes, epidemiology, global consequences, and control. Ecology Application 10:689-710.

Matthei, O. 1995. Manual de las malezas que crecen en Chile. Alfabeta Impresores. Santiago. Chile. 547 pp.

Matthei, O., C. Marticorena \& T.F. Stuessy. 1993. La flora adventicia del Archipiélago de Juan Fernández. Gayana Botánica (Chile) 50:69-102.

Martinic, M. 2000. Última Esperanza en el tiempo. Ediciones de la Universidad de Magallanes. Chile. $323 \mathrm{pp}$.

McKinney, M.L. 2002. Influence of settlement time, human population, park shape and age, visitation and roads on the number of alien plant species in protected areas in the USA. Diversity and Distributions 8:311-318.

Moore, D.M. \& R.N.P. GoodALl. 1977. La flora adventicia de Tierra del Fuego. Anales Instituto Patagonia, Punta Arenas (Chile) 8:263 - 274.

Moore, D.M. 1983. Flora of Tierra del Fuego. Oswestry, Saint Louis, EE.UU. 369 pp.

Ortega, I.M. \& W.L. Franklin. 1988. Feeding habitat utilization and preference by guanaco mole groups in the Chilean Patagonia. Revista Chilena de Historia Natural 61:209-216.
Pauchard, A. \& P. Alaback. 2004. Influence of elevation, land use, and landscape context on patterns of alien plant invasions along roadsides in protected areas of south-central Chile. Conservation Biology 18 (1):238-248.

PisAno, E. 1974. Estudios ecológicos de la región continental sur del área Andino-Patagónico. II: Contribución a la fitogeografía de la zona del "Parque Nacional Torres del Paine". Anales Instituto Patagonia, Punta Arenas (Chile), 5 (1-2):59-104.

Pisano, E., J.M. Henríquez, O. Dollenz, E. Domínguez, D.M. Moore, C. Venegas. \& V. Pérez. 1997. Establecimiento Biótico en Territorios en Proceso de Desglaciación en Fuego-Patagonia. Informe FONDECYT No 194113. 230 pp.

Pysek, P., D.M. Richardson, M. Rejmánek, G.L., Webster, M. Williamson \& J. Kirschner. 2004. Alien plants in checklists and floras: towards better communication between taxonomists and ecologists. Taxon 53(1): 131-143.

Rozzi, R., R. Charlin, S. IpPI \& O. Dollenz. 2004. Cabo de Hornos: un Parque Nacional libre de especies exóticas en el confín de América. Anales Instituto Patagonia (Chile) 32:55-62.

SкотtsвeRg, C. 1916. Die Vegetationsverhältnisse längs der Cordillera de los Andes S. von $41^{\circ} \mathrm{S}$. Br. Ein Beitrag zur Kenntnis der Vegetation in Chiloé, West-Patagonien, dem andinen Patagonien und Feuerland. K. Svenska Vetensk. Akad. Handl. 56(5):3-366.

Stohlgren, T.J., D.T. Barnett, \& J.T. Kartesz. 2001. Patterns of plant invasion: A case example in native species hotspots and rare habitats. Biological Invasions 3(1):37-50.

Stohlgren, T.J., G.W. Chong, L.D. Schell, K.A. RImar, Y. Otsuki, M. Lee, M.A. Kalkha, \& C.A. VILla. 2002. Assessing vulnerability to invasion by nonnative plant species at multiple scales. Environmental Management 29:566577.

Swenson, U., T.F. Stuessy, M. Baeza \& D.J. Crawford. 1997. New and historical plant introductions, and potencial pests in the Juan Fernández Islands. Chile. Pacific Science 51: 233-253. 